\title{
BMJ Open Increasing use of CT requested by emergency department physicians in tertiary hospitals in Western Australia 2003-2015: an analysis of linked administrative data
}

\author{
Susannah Maxwell, ${ }^{1}$ Ninh Thi Ha (D) , ${ }^{1}$ Max K Bulsara (D) , ${ }^{2,3}$ Jenny Doust, ${ }^{4}$ \\ Donald Mcrobbie, ${ }^{5}$ Peter O'Leary, ${ }^{1,6,7}$ John Slavotinek, ${ }^{8,9}$ Rachael Moorin (I) ${ }^{1,3}$
}

To cite: Maxwell S, Ha NT, Bulsara MK, et al. Increasing use of $\mathrm{CT}$ requested by emergency department physicians in tertiary hospitals in Western Australia 20032015: an analysis of linked administrative data. BMJ Open 2021;11:e043315. doi:10.1136/ bmjopen-2020-043315

- Prepublication history and supplemental material for this paper is available online. To view these files, please visit the journal online (http://dx.doi org $/ 10.1136 /$ bmjopen-2020043315).

Received 30 July 2020 Revised 16 January 2021 Accepted 17 February 2021

Check for updates

(C) Author(s) (or their employer(s)) 2021. Re-use permitted under CC BY-NC. No commercial re-use. See rights and permissions. Published by BMJ.

For numbered affiliations see end of article.

Correspondence to

Dr Ninh Thi Ha;

ninh.ha05@gmail.com

\section{ABSTRACT}

Objective This study aimed to examine trends in number of CT scans requested by tertiary emergency department (ED) physicians in Western Australia (WA) from 2003 to 2015 across broad demographic and presentation characteristics, anatomical areas and presented symptoms.

Design An observational cross-sectional study over study period from 2003 to 2015.

Setting Linked administrative health service data at individual level from WA.

Participants A total of 1666884 tertiary hospital ED presentations of people aged 18 years or older were included in this study

Main outcome measure Number of CT scans requested by tertiary ED physicians in an ED presentation.

Methods Poisson regression models were used to assess variation and trends in number of CT scans requested by ED physicians across demographic characteristics, clinical presentation characteristics and anatomical areas.

Results Over the entire study duration, 71 per 1000 ED episodes had a CT requested by tertiary ED physicians. Between 2003 and 2015, the rate of CT scanning almost doubled from 58 to 105 per 1000 ED presentations. After adjusted for all observed characteristics, the rate of CT scans showed a downward trend from 2009 to 2011 and subsequent increase. Males, older individuals, those attending ED as a result of pain, those with neurological symptoms or injury or with higher priority triage code were the most likely to have CT requested by tertiary ED physicians.

Conclusions Noticeable changes in the number of CTs requested by tertiary ED physicians corresponded to the time frame of major health reforms happening within WA and nationally.

\section{INTRODUCTION}

CT scanning is rapidly increasing globally over the last few decades. ${ }^{2}$ While some have reported this growth to have plateaued or slowed overall in recent years, CT use in the emergency setting has continued to

\section{Strengths and limitations of this study}

- In this study, we used a large linked administrative data for a length of 13 years to observe the trend in CT scan requested by emergency department (ED) physicians covering multiple healthcare reforms happening in Australia.

- Given availability of individual level data, we were able to assess the trend in CT scans with adjustment for variation in demographic and socioeconomic characteristics as well as clinical factors.

- Since not all secondary hospitals joined Western Australia Picture Archival Communication System (PACS) during the study period, this study was limited to assess CT scan in tertiary centres.

- The PACS data did not have information on the time that a CT scan was performed that prevented us from determining whether a CT scan was conducted while a patient was in ED, but we were able to determine the CT was requested by an ED physician in a tertiary hospital.

rise. ${ }^{34}$ Internationally increases in CT use in the emergency department (ED) have been reported in the USA, ${ }^{35-10}$ Canada, ${ }^{5}$ Taiwan $^{11}$ and Korea. $^{12} 13$

While an increase in CT use may reflect a response to changing patient characteristics (such as age) and/or the expansion in the indications for the use of CT, ${ }^{14}$ there are concerns that some of the increase could be inappropriate and lead to medical overuse, 'the provision of medical services for which the potential for harm exceeds the potential for benefit' ${ }^{15} 16$ Medical overuse in diagnostic imaging poses a significant problem for healthcare systems, contributing to rising costs and an increase in overdiagnosis and incidental findings that may expose patients to additional testing and associated unnecessary treatment, complications and psychological 
harm. ${ }^{1718}$ Furthermore, CT delivers a small but significant radiation exposure that has led to concerns regarding cumulative radiation dose, and associated cancer risk, for both individuals and populations. ${ }^{19}{ }^{20}$ Radiation exposure concerns have been addressed by guidelines and recommendations encouraging practitioners to work in line with the ALARA principle, that is to deliver radiation doses that are 'As Low As Reasonably Achievable'. ${ }^{21}$

In Australia, there is evidence of increasing CT use overall. ${ }^{22}{ }^{23}$ However, little is published about trends in CT use in ED in Australia or the characteristics of CT use in ED. ${ }^{22} 24{ }^{25}$ The number of CT scans billed through the Medicare Benefits Schedule (through subsidised private radiology) increased more than threefold between 1993/1994 and 2012/2013 (from 33 to 112 per annum per 1000 population)..$^{22}$

The value of examining linked-administrative data was recognised at the Academic Emergency Medicine consensus conference in $2015 .{ }^{26}$ While not a substitute for classical studies based on primary data collection, such data are invaluable in guiding targeted research questions to identify areas potential targeting for health planning and policy interventions. Western Australia (WA) has four tertiary hospitals (teaching hospitals), all located centrally in Perth. The universal health insurance scheme, Medicare, offers fee-free treatment to public patients in public hospitals that includes all tertiary hospital ED, ${ }^{27}$ although the funding mechanism is different between public hospitals and outside of public hospitals. While a previous study has examined the trend in CT use outside public hospital settings in $\mathrm{WA},{ }^{22}$ little has been published about the trend of CT use in public hospital settings, particularly in ED. This study aimed to describe trends in CT use initiated in tertiary hospital EDs in WA from 2003 to 2015, including by broad demographic and presentation characteristics, anatomical site and presented symptom using linked administrative data in WA.

\section{METHODS}

This was a retrospective observational cohort study using individual-level administrative health data linked through the WA Data Linkage Branch. Reporting follows the Reporting of studies Conducted using Observational Routinely-collected health Data (RECORD) guidelines. ${ }^{28}$

\section{Data sources}

The two datasets used in this study were:

1. WA Emergency Department Data Collection (EDDC) is a comprehensive data set of all presentations to EDs at public hospitals in WA. The data set consists of information related to episode of care within ED and demographic data required to be reported under the Australian Health Care Agreement. We used WA ED presentation records (2003-2015 inclusive) restricted to records in which the individual was aged 18 years and over with a WA residential postcode at the time of presentation. ED variables included demographic data (age, sex and postcode), the type of ED (tertiary or non-tertiary), presentation time and date, arrival code (ambulance or other), presentation type (unplanned presentation or other), triage code (the urgency of a patient's need for medical and nursing care), symptom codes collected on presentation at ED for the problem and concern that was the main reason for seeking healthcare, external cause of injury codes, destination and disposal (discharge) codes and sociodemographic data (remoteness index and Index of Relative Socioeconomic Disadvantage).

2. WA Picture Archival Communication System (PACS) database (2003-2015). PACS is a system to store and digitally transmit electronic images and clinically relevant reports. The PACS database includes all CT scans performed at tertiary hospitals and some scans performed in secondary hospitals in WA. PACS variables included the date, examination code (the code used by PACS to identify the CT scan undertaken; for this study, this code was used to determine the body region scanned), the referral source (ED, inpatient, outpatient clinic or external referral) and the provider (tertiary or other). As this study was interested in CT scanning in the tertiary hospital ED setting, we restricted the CT records to those where the referral source was ED, and the scan was undertaken at a tertiary hospital.

The two datasets were linked by the Data Linkage Branch in WA using data linkage techniques, which maximise possible matching while ensuring personal privacy. ${ }^{29}$ The linkage technique only uses demographic information for matching and then creates a unique scrambled person identifier for each individual in the data before providing the data to the research team. This allows records from the same individual within and across data sets to be determined without releasing identifiable personal information to researchers. Provider identifiers were not available in the data.

\section{Study population}

The study population included all unplanned tertiary ED episodes among those aged 18 plus years with a WA residential postcode at the time of presentation (2003-2015). A tertiary ED episode was defined as a single day with one or more tertiary ED presentations for an individual. Planned ED visits, such as a planned return visit, were excluded in order to capture true emergency presentations. Non-tertiary ED episodes were excluded from the analysis as secondary hospital scans are not consistently included in the PACS dataset (online supplemental appendix 1). Where an individual with a record of a tertiary ED presentation on a given day had multiple ED presentations on that day, the first tertiary presentation record was used as the data source.

\section{Outcome measures}

The main outcome measure is the number of CT episodes requested by a tertiary ED physician per 1000 
ED episodes. The CT episode associated with ED was defined as any CT record in the PACS dataset, which was requested by a tertiary ED physician and performed on the same or next day of the nearest ED presentation. CT episodes beyond this time frame were excluded for the purpose of this analysis.

We also present adjusted rates of CT examinations in different anatomical regions as listed in table 1 . The body regions under CT examinations were identified using the CT scan protocol codes in PACS data. The classification has been used in previous studies. ${ }^{3612}$

\section{Other measures}

Other measures were captured using information recorded in EDDC data. Demographic characteristics include sex (male/female) and age in years (18-44 years, $45-64$ years, $65-74$ years and 75 years or older). Social economic status was classified using socioeconomic index for area (SEIFA), which was developed by the Australian Bureau of Statistics that ranks areas in Australia according to relative socioeconomic advantage and disadvantage. Remoteness areas divide Australia into five classes of remoteness on the basis of a measure of relative access to services-Accessibility/remoteness Index of Australia (ARIA+). ${ }^{30}$ Triage code included resuscitation, emergency, urgent, semiurgent and non-urgent. Symptom category was classified based on $>3500$ ED symptom categories and classified into main symptom categories including pain, cardiovascular, gastrointestinal, injury, neurological, respiratory and others. ED episode presentation time was classified into three time-blocks: 00:0009:00, 09:00-17:00 and 17:00-00:00 to capture workload of EDs corresponding to daily work schedule of the general population. Other covariates included arrival type (ambulance/air and other), presentation date (weekend/public holiday or weekday) and presentation year.

Individuals with a non-tertiary ED presentation in the 24 hours prior to the primary tertiary presentation were flagged as having a previous non-tertiary ED presentation. As the time of service for the CT scan was not recorded, we adjusted for visits where a CT scan may have been undertaken as part of a prior associated non-tertiary ED presentation, potentially impacting the uptake of CT at the subsequent tertiary presentation.

\section{Statistical analysis}

The dataset aggregated by patient and visit characteristics was used to provide rates and adjusted rates of CT use per 1000 ED presentations over the period 2003-2015 and identify ED presentation characteristics associated with CT use. Multiple model specifications including Poisson regression, negative binominal regression (adjusted for overdispersion), zero-inflated Poisson regression with inflated constant (adjusted for excessive zeros in the count of CT episodes) and zero-inflated negative binominal regression with inflated constant were evaluated to select a suitable model. Both likelihood ratio test of dispersion parameter alpha and the information criteria including Bayes Information Criterion and Akaike Information Criterion suggested the negative binomial model for further analysis (online supplemental appendix 2). Independent covariates considered for the regression model included patient sociodemographics: sex, age group, SEIFA and remoteness index. Other covariates included triage code, main symptom category, year and time of presentation, arrival type, presentation type and weekend/public holiday or weekday, categories of which are shown in table 1 . As we only linked CT records that occurred on the same or next day to the ED episode, we included an ED episode presentation time variable to allow the model to adjust for variation in presentation time over the 13-year period. This accounts for the potential impact of differential follow-up time on the linkage of CT records over time. As an example, if all the ED episodes in year 1 occurred before 09:00, and all the ED episodes in year 13 occurred after 21:00, the year 1 ED episodes would have had between 12 and 24 hours more follow-up time than the latter year. The variable also works to adjust for changes in the use of CT by time-block.

To examine whether the trend of CT rate varied differently (ie, determine any effect modification) across characteristics of interest including age groups, sex, main symptom category and triage code, interaction terms for year and each characteristic were also included in the model. The predicted adjusted rates of CT use are presented between 2003 and 2015 for sex, age group, triage code and main symptom category using postestimation predictive margins.

CT rates by anatomical body region scanned were modelled using negative binomial regression to account for overdispersion. Rates of CT use by year for each of these anatomic regions are presented using postestimation predictive margins. ${ }^{31}$ The difference-in-differences approach was undertaken post hoc to examine whether there was a significant difference in the effect of the National Emergency Access Target programme (targeted in improving quality of care through the Four Hour Rule programme and activity-based funding implemented in Australia in January 2012) ${ }^{32}$ on the rate of CT use across sex, age group, triage code and main symptom category.

All data analyses were conducted using Stata SE V.15.

\section{RESULTS}

There were 1666884 unplanned tertiary ED episodes over the 13-year period, the characteristics of which are shown in table 1. These ED episodes linked to 118589 CT episodes, giving an overall unadjusted CT episode rate of $7.1 \%$.

The unadjusted trend of CT requested by tertiary ED physicians increased steadily from 2003 to 2015 from 58 to 105 per 1000 ED episodes. The adjusted trend of CT requested by tertiary ED physicians, adjusted by the variables shown in table 1, fluctuated between 2003 and 2011 with a very small overall reduction in CT (from 56 to 
Table 1 CT use and sociodemographic and ED presentation characteristics of ED episodes

ED episode characteristics

Total ( $\mathrm{n}=1666$ 884)

$\%$ of ED episodes

\begin{tabular}{|c|c|c|c|}
\hline \multirow[t]{8}{*}{ Outcome } & Any CT & 118589 & 7.1 \\
\hline & Head $\mathrm{CT}^{*}$ & 80772 & 4.9 \\
\hline & Abdomen pelvis $\mathrm{CT}^{*}$ & 22673 & 1.4 \\
\hline & Spine $\mathrm{CT}^{\star}$ (cervical/lumbar/thoracic) & 15634 & 0.9 \\
\hline & Chest $\mathrm{CT}^{*}$ & 10738 & 0.6 \\
\hline & Face $\mathrm{CT}^{*}$ & 3689 & 0.2 \\
\hline & Neck CT* & 3001 & 0.2 \\
\hline & Extremity $\mathrm{CT}^{\star}$ & 2626 & 0.2 \\
\hline \multicolumn{4}{|l|}{ Sociodemographic characteristics } \\
\hline Sex & Male & 831455 & 49.9 \\
\hline \multirow[t]{4}{*}{ Age (years) } & $18-44$ & 808472 & 48.5 \\
\hline & $45-64$ & 392855 & 23.6 \\
\hline & $65-74$ & 159914 & 9.6 \\
\hline & $75+$ & 305643 & 18.3 \\
\hline \multirow{5}{*}{$\begin{array}{l}\text { Socioeconomic index of relative } \\
\text { disadvantage }\end{array}$} & Least disadvantaged & 567971 & 34.1 \\
\hline & Less disadvantaged & 325040 & 19.5 \\
\hline & Moderate disadvantage & 372434 & 22.3 \\
\hline & High disadvantage & 220608 & 13.2 \\
\hline & Highest disadvantage & 158439 & 9.5 \\
\hline \multirow[t]{4}{*}{ Remoteness index } & Highly accessible (major city) & 1572748 & 94.4 \\
\hline & Accessible & 33442 & 2.0 \\
\hline & Moderately accessible & 23472 & 1.4 \\
\hline & Remote/very remote & 21727 & 1.3 \\
\hline \multicolumn{4}{|l|}{ ED presentation characteristics } \\
\hline \multirow[t]{5}{*}{ Triage code } & Resuscitation & 30429 & 1.8 \\
\hline & Emergency & 315786 & 18.9 \\
\hline & Urgent & 600942 & 36.1 \\
\hline & Semiurgent & 587095 & 35.2 \\
\hline & Non urgent & 132632 & 8.0 \\
\hline \multirow[t]{2}{*}{ Arrival } & Ambulance/air & 651401 & 39.1 \\
\hline & Other & 1015483 & 60.9 \\
\hline \multirow[t]{2}{*}{ Weekend or public holiday } & Weekday & 1112724 & 66.8 \\
\hline & Weekend/public holiday & 554160 & 33.2 \\
\hline \multirow[t]{7}{*}{ Main symptom category } & Pain & 509133 & 30.5 \\
\hline & Injury & 322138 & 19.3 \\
\hline & Neurological & 157222 & 9.4 \\
\hline & Respiratory & 97453 & 5.9 \\
\hline & Gastrointestinal & 85958 & 5.2 \\
\hline & Cardiovascular & 44515 & 2.7 \\
\hline & Other & 450465 & 27.0 \\
\hline \multirow[t]{3}{*}{ Presentation time } & 09:00-17:00 & 673620 & 40.4 \\
\hline & $17: 00-00: 00$ & 530926 & 31.9 \\
\hline & 00:00-09:00 & 462338 & 27.7 \\
\hline
\end{tabular}

*Not mutually exclusive.

ED, emergency department. 


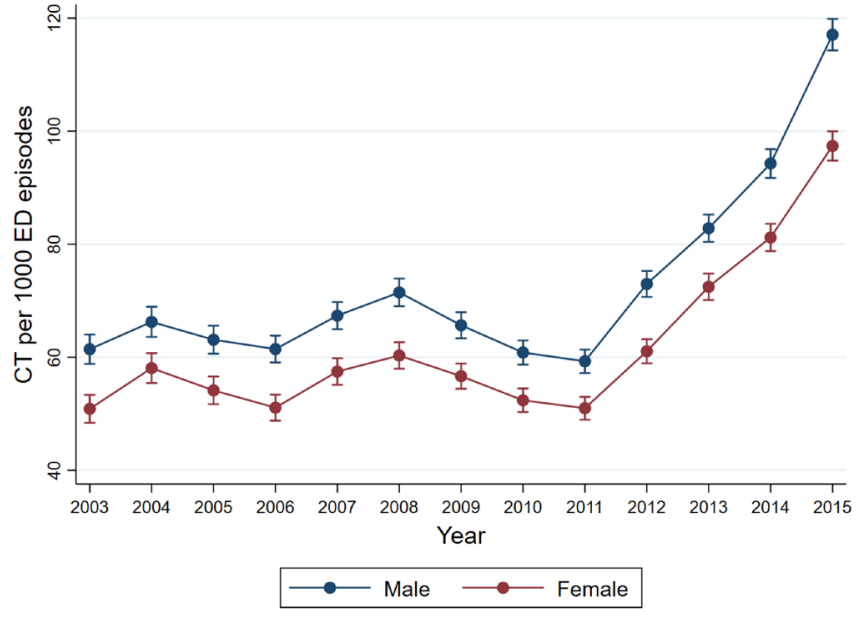

Figure 1 Adjusted CT per 1000 ED episodes by sex with 95\% Cls, 2003-2015. ED, emergency department.

55 adjusted per 1000 ED episodes); however, from 2011 rates rose consistently, almost doubling by 2015 (from 55 to 107 per 1000 ED episodes). Rates by sex and age over years with 95\% CIs are shown in figures 1 and 2 with similar increased rates after 2011. Males (figure 1), those in the highest triage categories (figure 3) and those presenting with neurological symptoms or with pain or injury (figure 4) were the most likely to have a CT episode. Higher age was associated with higher CT episodes. The older age groups also had larger proportionate increases in their rate of CT use overtime with 1.4fold, 1.84-fold, 2.0-fold and 2.5-fold increases in the rate of CT for 18-44, 45-64, 64-74 and 75+ year olds, respectively, between 2003 and 2015. While younger individuals were consistently less likely to have CT throughout the study period, the older age groups did not become well differentiated from each other until after 2012 (figure 2 and details in online supplemental appendix 3 ). The post hoc analysis using difference-in-differences approach indicated that the expected mean change in the CT use from before to after the national health reform in 2012

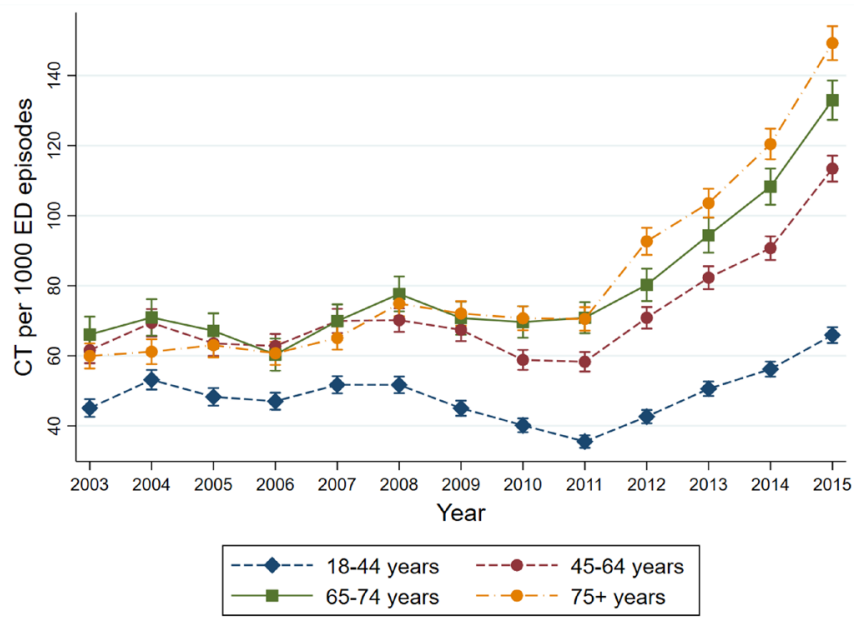

Figure 2 Adjusted CT per 1000 ED episodes by age with 95\% Cls 2003-2015. ED, emergency department.

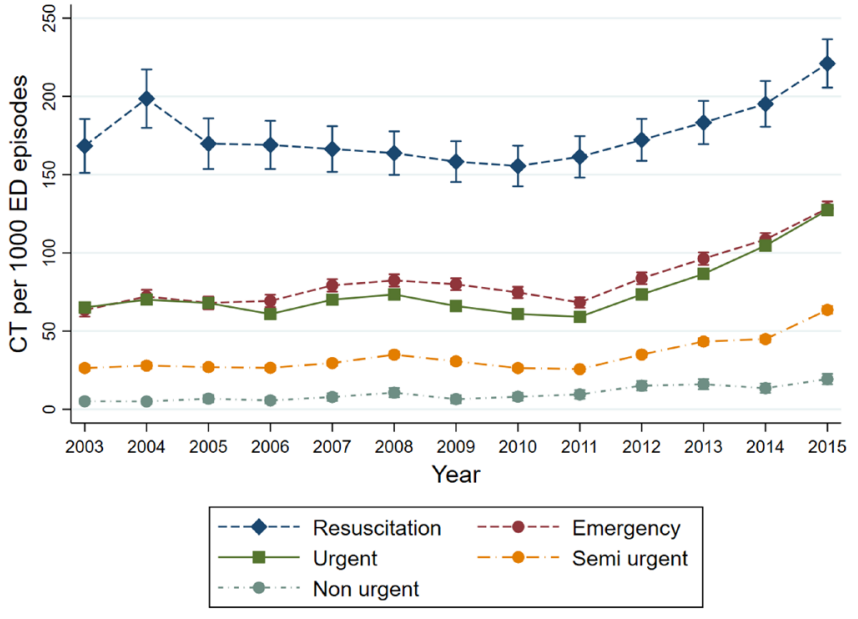

Figure 3 Adjusted CT per 1000 ED episodes by triage code with 95\% Cls 2003-2015. ED, emergency department.

was significantly higher in the older age groups compared with the youngest age group with IRR was 1.19, 1.29 and 1.49 for 45-64 years, 65-74 years and 75+ years, respectively (details of the effect is presented in the online supplemental appendix 4). Similarly, we also observed a significant difference in the effect of the policy across symptom category (vs pain) and triage code (vs resuscitation). However, there was no difference in the effect of the policy between male and female after adjusted for all observed demographic and clinical characteristics. Remoteness (ie, major city vs regional) and socioeconomic area were not associated with variation in CT use (online supplemental appendix 3). The most commonly scanned anatomic areas were head, abdomen/pelvis, spine and chest that were consistent over years (figure 5).

\section{DISCUSSION}

Using linked administrative data to evaluate the trends in CT requested by tertiary ED physicians, this study shows

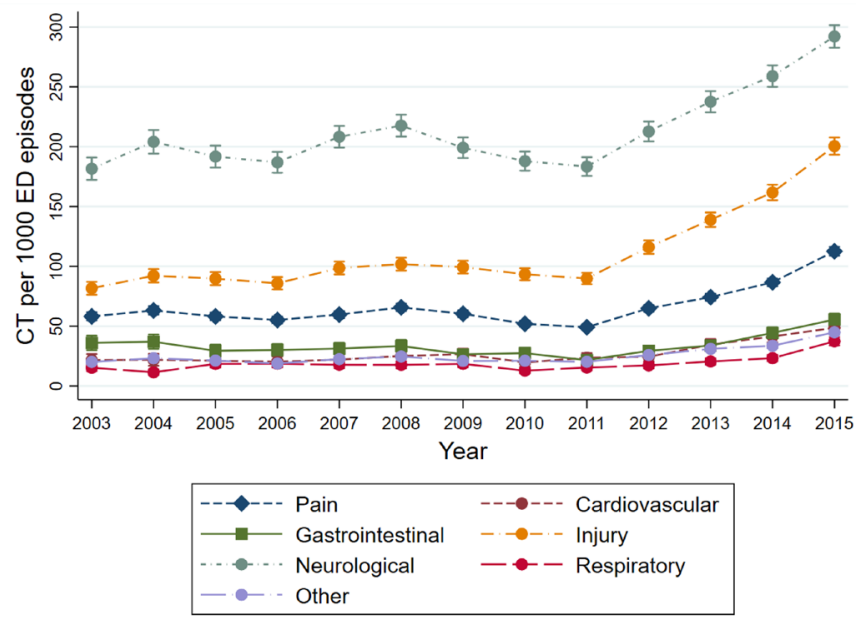

Figure 4 Adjusted CT per 1000 ED episodes by symptom category with 95\% Cls 2003-2015. ED, emergency department. 


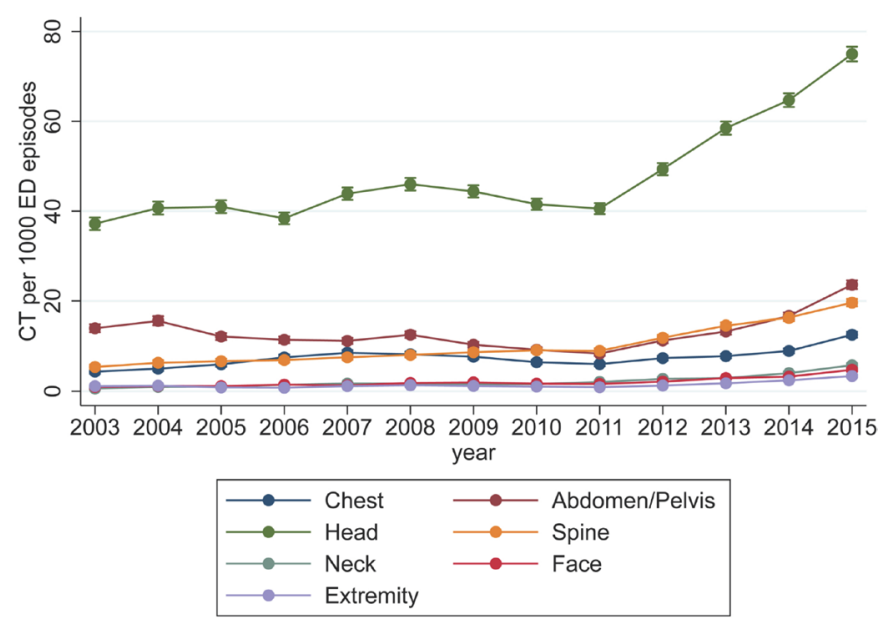

Figure 5 Adjusted CT per $1000 \mathrm{ED}$ episodes by anatomic region scanned with 95\% Cls 2003-2015. ED, emergency department.

a large increase in the rate per 1000 of CTs requested by tertiary ED physicians in WA, particularly from 2011 onwards, with this increase most pronounced for head CT. Overall, the rate of CTs requested by tertiary ED physicians increased by $80 \%$, from 58 to 105 per 1000 ED episodes (unadjusted 2003-2015), consistent with general trends reported previously, ${ }^{36912}$ although many of these were conducted in a single institution. A study using an administrative claim database in the USA found that the use of CT scans per 1000 ED visits increased about $60 \%$, but the rate is much higher in the US setting, from 153 CTs in 2005 to 245 CTs in $2013 .{ }^{6}$

Our results, adjusted for clinical and sociodemographic characteristics, suggest that the change in CT use has been driven by clinical practice change, rather than changes in the characteristics of those attending ED. The drivers of these changes across the time period-the slight downwards trend from 2009 to 2011 and subsequent increase-are likely to be complex and inter-related. ${ }^{18} 33$ While specific investigation on clinical factors is outside this study scope, exploring relevant health policies may help to better understand potential drivers of the growth of CT and direct future research.

Two major health reforms occurred during the study period. The Four Hour Rule Program was initiated in 2009 in WA and the National Health Reform (NHR) through activity-based funding was implemented at the end of 2011 in Australia. These may explain the trends observed in our study. The Four Hour Rule Program (FHR) was first initiated in $\mathrm{UK}^{34}{ }^{35}$ and then adopted in WA to be implemented in all metropolitan hospitals in November $2009 .^{34}$ While the programme showed an improvement in ED efficiency and patient experience, the programme review in WA indicated a clear shift of radiology activity to the wards. ${ }^{35}$ The number of all radiology examinations referred from EDs decreased by 11\% from March 2009 to September 2011, while the referrals from the wards increased by $191 \%{ }^{36}$ This is consistent with the downward trend in the rate of CT referred by ED observed in our data in the same period (2009-2011).

From August 2011 onwards, the NHR was implemented in all state through the use of activity-based funding model (ABF). ${ }^{37}$ The reform replaced the previous funding scheme, which was based on historical number of patient presentations and political consideration that had limited relation to real cost associated with delivering care.$^{38}$ Under the ABF model, the costs of delivering hospital services including ED are based on the volumes and price paid for type of services delivered to patient that gives hospitals an incentive to provide services more efficiently. ${ }^{37} 38$ The FHR programme was also incorporated into the NHR under the National Emergency Access Target (NEAT) implemented across Australia since January $2012 .{ }^{32}$ State and territory governments are given significant financial incentives from the federal government if the 4-hour rule is achieved in $90 \%$ patients presenting at ED by $2015 .^{32}$ The multiple reforms targeting processes in both ED and its interface inpatient unit have broad impacts in healthcare. Studies evaluating the impact of the 2012 reform showed a significant improvement in timeliness of care for patients presenting at ED and reduce in length of stay in ED, patient mortality and access bed block. ${ }^{3435} 3940$ However, a systematic review on the impact of $\mathrm{ABF}$ suggested that the $\mathrm{ABF}$ model could also cause unintended adverse consequences such as increase readmissions and severity of illness. ${ }^{41}$ Similarly, the NEAT may result in increasing pressure on ED physicians to achieve the 4-hour rule target. ${ }^{42} \mathrm{~A}$ study conducted in a tertiary hospital in Australia found an increase up to $61 \%$ in the total number of imaging requests following the introduction of the 2011 reform compared with the prereform period. ${ }^{43}$ Interestingly, this study indicated that the trend was towards more requests in CT and less radiography while other imaging techniques remained unchanged. ${ }^{43}$ This finding is in line with our results, which indicated a significant increase in CT use associated with ED across age, sex, diagnostic groups and triage categories. While our main objective is not to evaluate the impact of the NHR reform in 2011, our result may provide a valuable information to aid our understanding in the use of diagnostic imaging in ED under the different health reforms. Our study may direct future research to examine whether the health reforms may cause the growth of CT use in tertiary care both in $\mathrm{ED}$ and its inpatient interface to inform policy making.

In addition, advances in CT technology and staffing adjustments during this time period may also have a role in the growth of CT use in ED after 2011. ${ }^{12}$ Increased quality of CT images, such as faster higher slice count CTs, with subsequent improved accuracy and quality of diagnostic information, has led to an expansion of the indications for CT and has contributed to increased CT rates. Other factors that may impact CT use include tolerance of diagnostic uncertainty, ${ }^{44}$ or the perception of harms and benefits, which may be altered as a result of awareness raising initiatives or technological advances. 
Regarding the latter, it is plausible that the introduction of iterative reconstruction software-known as 'low dose CT' - for all Philips Healthcare CT equipment in WA public hospitals in 2011/2012 played a role in increasing CT use from that time, if the availability of 'low dose CT' had led to the perception among practitioners and/or patients that radiation dose had decreased significantly, with harms reducing commensurately. ${ }^{45}$ Iterative reconstruction software reduces image noise and affords the opportunity to reduce radiation dose; however, to fully realise this potential, practitioners must forego the opportunity to further improve image quality. Of concern is that postimplementation evaluation of iterative reconstruction software in some WA public hospitals demonstrated that in clinical practice, radiation dose varied by protocol and had not been reduced to the extent seen in experimental studies. ${ }^{45}$

The greater effect modification of age group on CT rates post-2012 may reflect changes among those presenting at ED that are not captured in our data, or changing recommendations for CT in particular populations. This finding is in line with literature-examined CT rates in ED in the same study period. ${ }^{3646}$ This change could also be in part due to increased practitioner awareness of the decreasing harms of CT as individuals get older. Harms of CT are greater for younger individuals who have higher risk from radiation exposure due to increased tissue sensitivity and greater postexposure life expectancy.

Our study observed substantial differences in the rate of CT between male and female in with male consistently had a higher rate of CT than female across the study period after adjusted for all observed demographic and clinical characteristics. This can be explained by the characteristics of the population presenting at tertiary ED which had injury and neurological symptoms of which $60 \%$ being male, being as the most common symptom, after pain. Our finding is in line with other studies that examined the use of CT in ED using Medicare Advantage enrolees in USA, ${ }^{6}$ a tertiary care centre ${ }^{12}$ and a mixed of both tertiary and secondary care ED. ${ }^{47}$ However, another study found an opposite result event using data from a tertiary setting for population aged 18 years or older. ${ }^{3}$

The most commonly scanned anatomic areas in our study-head, abdomen/pelvis-were also consistent with other studies. ${ }^{9} 12$ The pronounced increase in head CT scan warrants further research to investigate specific head CT and head CT-related protocols or CT use in specific clinical conditions such as injury and neurological conditions to identify potential medical overuse and to inform planning and management. In addition, the NHR reform in the end 2011 may create a potential financial incentive to increasing the use of $\mathrm{CT}$ in $\mathrm{ED}$, therefore, further research focusing on evaluating the impact of the activity based funding on the use of CT in ED is warranted.

\section{Strengths and limitations}

A major strength of our study is the size of the cohort, capturing ED presentations over 13 years at all tertiary hospitals in WA, and the inclusion of sociodemographic and clinical characteristics in the dataset. The data provide an opportunity to evaluate the trend in CT scan use at the population level over time. The availability of sociodemographic and clinical characteristics in the data allows to evaluate of the use of CT scan across different population as well as to adjust for the variation of the characteristics which may impact on the growth of CT scan.

Despite the richness of these data, the analysis had some limitations, inherent in the use of linked administrative datasets for health services research. We did not have consistently available CT data for secondary hospitals, requiring us to limit this study to tertiary centres, the impact of which on our results was taken into account by adjusting for a previous secondary presentation. Future research with data linked across different health sectors would provide opportunities to explore cost diversion for imaging, from general practitioner (GP) to hospitals, secondary to tertiary and/or from ED to GP and support a comprehensive monitoring CT use in populations and guiding policy making.

Our data cannot tell us the exact clinical decision making involved in the decision to request CT or not as we do not have information on the use of other diagnostic imaging modalities or a complete picture of the presentation of the patient. In addition, the PACS data did not have information on the time that a CT scan was performed, which prevented us from determining whether a CT scan was conducted while a patient was in ED or after being admitted to a ward. However, we were able to determine the CT was requested by an ED physician in a tertiary hospital.

A further limitation of our research is the time frame of the data. Due to the disaggregation of the custodianship of Picture Archiving Communications data in early 2016 in WA, we are unable to access data for the most recent years. Unfortunately, this means that we have minimal follow-up time to assess any impact of Emergency Department Choosing Wisely messages for diagnostic imaging-implemented in Australia in late April 2015. The Choosing Wisely campaign-first introduced in the USA in 2012-is an initiative to reduce the use of 'tests, treatments and procedures that provide no benefit or, some cases, lead to harm', and in April 2015, three choosing wisely messages were introduced in Australia relating to CT use in the emergency setting. ${ }^{48}$

\section{CONCLUSION}

While CT scan referred by ED shows relatively little change to 2009, there is a slight downwards trend to 2011 and subsequent increase. While the increase cannot be attributed solely to changes in ED presentation characteristics, this may be a result of major health reforms happening within WA and nationally (FHR and $\mathrm{ABF}$ ) given the noticeable changes observed in the data corresponding to the time frame of the two health reforms. Further targeted, in-depth examination in particular 
areas is required to determine the characteristics of tertiary (and other) ED presentations, if any, that are associated with the growth of CT use.

\section{Author affiliations}

${ }^{1}$ Health Economics and Data Analytics, Curtin University Bentley Campus, Perth, Western Australia, Australia

${ }^{2}$ Institute for Health and Rehabilitation Research, University of Notre Dame, Fremantle, Western Australia, Australia

${ }^{3}$ Centre for Health Services Research, School of Population and Global Health, Faculty of Medicine, Dentistry and Health Sciences, The University of Western Australia, Crawley, Western Australia, Australia

${ }^{4}$ Centre for Longitudinal and Life Course Research, School of Public Health, Faculty of Medicine, University of Queensland, Brisbane, Queensland, Australia

${ }^{5}$ School of Physical Sciences, University of Adelaide, Adelaide, South Australia, Australia

${ }^{6}$ Obstetrics and Gynaecology Medical School, Faculty of Health and Medical Sciences, The University of Western Australia, Perth, Western Australia, Australia

${ }^{7}$ PathWest Laboratory Medicine, QE2 Medical Centre, Nedlands, Western Australia, Australia

${ }^{8}$ Flinders Medical Centre, Bedford Park, South Australia, Australia

${ }^{9}$ College of Medicine and Public Health, Flinders University, Adelaide, South Australia, Australia

Acknowledgements We would like to thank the National Health and Medical Research Council for supporting this work. We would also like to acknowledge the Western Australian Data Linkage Branch and the data custodians of the WA Emergency Department Data Collection and the Picture Archiving Communications System data, as well as the individuals whose data enabled this study. We also acknowledge the contribution of Professor Richard Fox for his work in the conceptualisation and editing of this manuscript and Mr John Stubbs for his contribution as a consumer representative.

Contributors All authors contributed to the study design and concept. RM, SM and NTH contributed to the acquisition of data. RM, MKB, JD, DM, PO, JS, SM and NTH contributed to the analysis and interpretation of the data. RM, SM and NTH contributed to drafting of the manuscript. RM, JD, DM, PO, JS, SM and NTH contributed to critical revision of the manuscript for important intellectual content. RM, SM, NTH and MKB contributed to statistical expertise. RM, MKB, JD, DM, PO and JS secured funding for the study.

Funding This study was funded with an Australian National Health and Medical Research Council grant, project grant APP1144573.

Competing interests The institutions of RM, NTH, PO, SM, MKB and DM received grant funding from the National Medical Research Council of Australia for investigator-initiated research.

Patient consent for publication Not required.

Ethics approval Ethics approval was provided by the Curtin University Human Research Ethics Committee (SMEC-80-10) and the Western Australian Department of Health Human Research Ethics Committee (2011/97).

Provenance and peer review Not commissioned; externally peer reviewed.

Data availability statement Data may be obtained from a third party and are not publicly available. The data that support the findings of this study are available through application to the Western Australian Data Linkage Branch and the Western Australian Department of Health Human Ethics Research Committee. Restrictions apply to the availability of these data. Researchers wishing to access the datasets used in this study should refer to the WA Data Linkage application process (https:// www.datalinkage-wa.org.au/access-and-application).

Supplemental material This content has been supplied by the author(s). It has not been vetted by BMJ Publishing Group Limited (BMJ) and may not have been peer-reviewed. Any opinions or recommendations discussed are solely those of the author(s) and are not endorsed by BMJ. BMJ disclaims all liability and responsibility arising from any reliance placed on the content. Where the content includes any translated material, BMJ does not warrant the accuracy and reliability of the translations (including but not limited to local regulations, clinical guidelines, terminology, drug names and drug dosages), and is not responsible for any error and/or omissions arising from translation and adaptation or otherwise.
Open access This is an open access article distributed in accordance with the Creative Commons Attribution Non Commercial (CC BY-NC 4.0) license, which permits others to distribute, remix, adapt, build upon this work non-commercially, and license their derivative works on different terms, provided the original work is properly cited, appropriate credit is given, any changes made indicated, and the use is non-commercial. See: http://creativecommons.org/licenses/by-nc/4.0/.

ORCID iDs

Ninh Thi Ha http://orcid.org/0000-0002-2789-5604

Max K Bulsara http://orcid.org/0000-0002-8033-6123

Rachael Moorin http://orcid.org/0000-0001-8742-7151

\section{REFERENCES}

1 Brady Z, Forsythe AV, Mathews JD. The changing use of pediatric CT in Australia. Pediatr Radiol 2016;46:1199-208.

2 Levin DC, Parker L, Rao VM. Recent trends in imaging use in hospital settings: implications for future planning. J Am Coll Radiol 2017;14:331-6.

3 Bellolio MF, Bellew SD, Sangaralingham LR, et al. Access to primary care and computed tomography use in the emergency department. BMC Health Serv Res 2018;18:154.

4 Levin DC, Rao VM, Parker L, et al. Continued growth in emergency department imaging is bucking the overall trends. J Am Coll Radiol 2014;11:1044-7.

5 Berdahl CT, Vermeulen MJ, Larson DB, et al. Emergency department computed tomography utilization in the United States and Canada. Ann Emerg Med 2013;62:486-94.

6 Bellolio MF, Heien HC, Sangaralingham LR, et al. Increased computed tomography utilization in the emergency department and its association with hospital admission. West J Emerg Med 2017;18:835-45.

7 Broder J, Warshauer DM. Increasing utilization of computed tomography in the adult emergency department, 2000-2005. Emerg Radiol 2006;13:25-30.

8 Hess EP, Haas LR, Shah ND, et al. Trends in computed tomography utilization rates: a longitudinal practice-based study. J Patient Saf 2014;10:52-8.

9 Lee J, Kirschner J, Pawa S, et al. Computed tomography use in the adult emergency department of an academic urban hospital from 2001 to 2007. Ann Emerg Med 2010;56:591-6.

10 Prabhakar AM, Gottumukkala RV, Hemingway J, et al. Increasing utilization of emergency department neuroimaging in Medicare beneficiaries from 1994 to 2015. Am J Emerg Med 2018;36:680-3.

11 Hu S-Y, Hsieh M-S, Lin M-Y, et al. Trends of CT utilisation in an emergency department in Taiwan: a 5-year retrospective study. BMJ Open 2016;6:e010973.

12 Ahn S, Kim WY, Lim KS, et al. Advanced radiology utilization in a tertiary care emergency department from 2001 to 2010. PLoS One 2014:9:e112650.

13 Oh HY, Kim EY, Cho J, et al. Trends of CT use in the adult emergency department in a tertiary academic hospital of Korea during 20012010. Korean J Radiol 2012;13:536-40.

14 Pelc NJ. Recent and future directions in CT imaging. Ann Biomed Eng 2014;42:260-8.

15 Weidman EK, Loftus ML. The Consequences of Inappropriate Use of Emergency Imaging. In: Kelly A, Cronin P, Puig S, et al, eds. Evidence-Based emergency imaging: optimizing diagnostic imaging of patients in the emergency care setting. Cham: Springer International Publishing, 2018: 37-46.

16 Brownlee S, Chalkidou K, Doust J, et al. Evidence for overuse of medical services around the world. Lancet 2017;390:156-68.

17 O'Sullivan JW, Muntinga T, Grigg S, et al. Prevalence and outcomes of incidental imaging findings: umbrella review. BMJ 2018;361:k2387.

18 Baloescu C. Diagnostic Imaging in Emergency Medicine: How Much Is Too Much? Ann Emerg Med 2018;72:637-43.

19 Griffey RT, Sodickson A. Cumulative radiation exposure and cancer risk estimates in emergency department patients undergoing repeat or multiple CT. AJR Am J Roentgenol 2009;192:887-92.

20 Berrington de González A, Mahesh M, Kim K-P, et al. Projected cancer risks from computed tomographic scans performed in the United States in 2007. Arch Intern Med 2009;169:2071-7.

21 Costello JE, Cecava ND, Tucker JE, et al. Ct radiation dose: current controversies and dose reduction strategies. AJR Am J Roentgenol 2013;201:1283-90.

22 Wright CM, Bulsara MK, Norman R, et al. Increase in computed tomography in Australia driven mainly by practice change: a decomposition analysis. Health Policy 2017;121:823-9. 
23 Gibson DA, Moorin RE, Semmens J, et al. The disproportionate risk burden of CT scanning on females and younger adults in Australia: a retrospective cohort study. Aust N Z J Public Health 2014;38:441-8.

24 Street M, Brady Z, Van Every B, et al. Radiation exposure and the justification of computed tomography scanning in an Australian hospital emergency department. Intern Med J 2009;39:713-9.

25 Goergen SK, Grimm J, Paul E, et al. Audit of demand for after-hours CT scanning services in RANZCR-accredited training departments. $J$ Med Imaging Radiat Oncol 2016;60:35-46.

26 Kuehl DR, Berdahl CT, Jackson TD, et al. Advancing the use of administrative data for emergency department diagnostic imaging research. Acad Emerg Med 2015;22:1417-26.

27 Australian Government Australian Institute of Health and Welfare. Australia's Health 2016, 2016. Available: https://www.aihw.gov.au/ getmedia/9844cefb-7745-4dd8-9ee2-f4d1c3d6a727/19787-AH16. pdf.aspx

28 Benchimol El, Smeeth L, Guttmann A, et al. The reporting of studies conducted using observational Routinely-collected health data (record) statement. PLoS Med 2015;12:e1001885.

29 Data Linkage Western Australia. Data Linkage Branch Services Linkage Data Linkage Western Australia, 2021. Available: https:// www.datalinkage-wa.org.au/dlb-services/linkage/

30 Australian Bureau of Statistics. 1270.0.55.005 - Australian Statistical Geography Standard (ASGS): Volume 5 - Remoteness Structure, July 2016, 2018. Available: https://www.abs.gov.au/ausstats/abs@.nsf/ $\mathrm{mf} / 1270.0 .55 .005$ ? OpenDocument

31 Williams R. Using the margins command to estimate and interpret adjusted predictions and marginal effects. Stata $J$ 2012;12:308-31.

32 Prang K-H, Canaway R, Bismark M, et al. The impact of Australian healthcare reforms on emergency department time-based process outcomes: an interrupted time series study. PLoS One 2018;13:e0209043.

33 Government of Western Australia - Department of Health. What is the national ABF program? Western Australia: Government of Western Australia - Department of Health. Available: https://ww2.health.wa. gov.au/Our-performance/Activity-based-funding-and-management/ What-is-the-national-ABF-program

34 Stokes B. Four hour rule program progress and issues review. Perth, Western Australia: Department of Health, 2011.
$35 \mathrm{Ngo} \mathrm{H}$, Forero R, Mountain D, et al. Impact of the four-hour rule in Western Australian hospitals: trend analysis of a large record linkage study 2002-2013. PLoS One 2018;13:e0193902-e.

36 Hughes G. The four hour target in Western Australia: a progress report. Emerg Med J 2012;29:526-7.

37 Solomon S. Health reform and activity-based funding. Med J Aust 2014;200:564.

38 Wylie K, Bell A, FitzGerald G. The implications of activity based funding for emergency departments: a comprehensive literature review. Brisbane, Australia: Queensland University of Technology, 2014.

39 Lowthian J, Curtis A, Straney L, et al. Redesigning emergency patient flow with timely quality care at the Alfred. Emerg Med Australas 2015;27:35-41.

40 Sullivan CM, Staib A, Flores J, et al. Aiming to be neat: safely improving and sustaining access to emergency care in a tertiary referral hospital. Aust Health Rev 2014;38:564-74.

41 Palmer KS, Agoritsas T, Martin D, et al. Activity-Based funding of hospitals and its impact on mortality, readmission, discharge destination, severity of illness, and volume of care: a systematic review and meta-analysis. PLoS One 2014;9:e109975.

42 Reddy $\mathrm{S}$, Jones $\mathrm{P}$, Shanthanna $\mathrm{H}$, et al. A systematic review of the impact of healthcare reforms on access to emergency department and elective surgery services: 1994-2014. Int J Health Serv 2018;48:81-105.

43 Tse $\mathrm{R}$, Thompson N, Moscova M, et al. Do delays in radiology lead to breaches in the 4-hour rule? Clin Radiol 2016;71:523-31.

44 Lawton R, Robinson O, Harrison R, et al. Are more experienced clinicians better able to tolerate uncertainty and manage risks? A vignette study of doctors in three NHS emergency departments in England. BMJ Qual Saf 2019;28:382-8.

45 Moorin RE, Gibson DAJ, Forsyth RK, et al. The impact of iterative reconstruction on computed tomography radiation dosimetry: evaluation in a routine clinical setting. PLoS One 2015;10:e0138329.

46 Juliusson G, Thorvaldsdottir B, Kristjansson JM, et al. Diagnostic imaging trends in the emergency department: an extensive singlecenter experience. Acta Radiol Open 2019;8:205846011986040.

47 Li C-J, Syue Y-J, Lin Y-R, et al. Influence of CT utilisation on patient flow in the emergency department: a retrospective 1-year cohort study. BMJ Open 2016;6:e010815.

48 NPS MedicineWise. Choosing wisely Australia, 2019. 